\title{
Estilo comportamental al inicio del segundo año de vida: estudio retrospectivo en escolares afectados de trastorno por déficit de atención e hiperactividad
}

\author{
S. Magallón ${ }^{a}$, N. Crespo-Eguílaz ${ }^{a} *$, M. Ecay ${ }^{a}$, M.L. Poch-Olivéb y J. Narbona ${ }^{a}$ \\ a Unidad de Neurología Pediátrica, Departamento de Pediatría, Clínica Universitaria de Navarra, Departamento de \\ Educación, Universidad de Navarra, Pamplona, España \\ bUnidad de Neuropediatría, Servicio de Pediatría, Hospital San Pedro, Logroño, España
}

Recibido el 11 de diciembre de 2008; aceptado el 21 de enero de 2009

Disponible en Internet el 7 de mayo de 2009

\author{
PALABRAS CLAVE \\ Déficit de atención; \\ Estilo comportamen- \\ tal; \\ Juego; \\ Niñez temprana; \\ Predicción; \\ Temperamento \\ infantil; \\ TDAH
}

\begin{abstract}
Resumen
Objetivos: Estudiar la posible relación entre el perfil comportamental de escolares afectos de trastorno por déficit de atención e hiperactividad (TDAH) y el estilo de conducta que poseían al inicio del segundo año de vida.

Sujetos y métodos: Los padres de 50 niños afectos de TDAH y los padres de 30 niños controles contestaron una versión española del TBQ (Toddler Behaviour Questionnaire) acerca de sus percepciones retrospectivas de la conducta de sus hijos al inicio del segundo año de vida. Los ítems del TBQ se agruparon mediante un análisis factorial. Se estudiaron las diferencias (t-Student) de las puntuaciones del TBQ entre ambos grupos y se aplicó un análisis de correlación múltiple entre las puntuaciones en el TBQ y en la escala del Manual Diagnóstico y Estadístico de los Trastornos Mentales IV del TDAH.

Resultados: Según la percepción de sus padres, los niños afectos de TDAH (como grupo) habían mostrado al inicio del segundo año un estilo de conducta diferente al de los niños del grupo control $(p<0,05)$; estas diferencias se daban en las siguientes dimensiones de la conducta: regularidad, humor y estabilidad durante el juego.

Conclusiones: Aunque los resultados del presente estudio deben considerarse con precaución, parecen mostrar que desde el quinto trimestre de vida es diferenciable un particular estilo de conducta en lo tocante a regularidad, humor y estabilidad durante el juego, que puede indicar riesgo de TDAH en edades posteriores. Este estilo de conducta debe tenerse en cuenta en la educación temprana y ser objeto de estudios prospectivos. (c) 2008 Asociación Española de Pediatría. Publicado por Elsevier España, S.L. Todos los derechos reservados.
\end{abstract}

\footnotetext{
*Autor para correspondencia.

Correo electrónico: necrespo@unav.es
}

1695-4033/\$ - see front matter ๔ 2008 Asociación Española de Pediatría. Publicado por Elsevier España, S.L. Todos los derechos reservados. doi:10.1016/j.anpedi.2009.01.018 
KEYWORDS

Attention Deficit; Behavioural style; Play; Toddlers; Prediction; Early temperament; ADHD

\section{Children with ADHD: A retrospective description of their behavioural features as toddlers}

\begin{abstract}
Objectives: To study the relationship between behavioural profile of children suffering from Attention Deficit Hyperactivity Disorder (ADHD) and the previous behavioural style of these patients as toddlers.

Subjects and methods: We asked the parents of 50 schoolchildren with ADHD, and those of 30 controls, to fill in a Spanish version of the Toddler Behaviour Questionnaire (TBQ) from their retrospective perception of their children's behaviour as toddlers. TBQ items were grouped by factor analysis; t-Student between the scores of both groups and a multiple correlation analysis of TBQ and DSM-IV-ADHD-RS in each of the groups were used.

Results: Children in the ADHD group were reported by parents to have had a different toddler behavioural profile in comparison to that of control children $(P<0.05)$. These differences were associated with adapting to new environments, mood, regularity and stability of play behaviour. A correlation was found between behavioural profile in DSM-IVADHD- RS and TBQ.

Conclusions: The results of this study should be interpreted with caution. However, they suggest that in the fifth trimester of life a particular behavioural style as regards regularity, stability of play, and mood, could indicate a risk of developing ADHD in the future. This behavioural style should be taken into consideration in rearing and early education prospective studies.

(c) 2008 Asociación Española de Pediatría. Published by Elsevier España, S.L. All rights reserved.
\end{abstract}

\section{Introducción}

La atención puede definirse como la actualización de la consciencia. Los niños con dificultades para focalizar, mantener la atención y autorregular su conducta a edades tempranas pueden beneficiarse de recursos educativoterapéuticos si estas dificultades son detectadas ${ }^{1-3}$. De aquí la utilidad de definir las manifestaciones de conductas alejadas de la normalidad en cuanto a la atención en la infancia temprana. El objetivo de esta investigación es estudiar las características estables de la conducta todavía poco influidas por la educación a una edad tan prematura como de 12 a 15 meses; se limita a los niños en los que más tarde se desarrollará el fenotipo conductual del trastorno por déficit de atención e hiperactividad (TDAH). Una de las premisas de las que se parte es que este trastorno depende en gran medida de factores genéticos y que, por tanto, puede traducirse en un particular estilo de conducta durante la niñez temprana. Así, se considera que la capacidad para focalizar y mantener la atención es un rasgo temperamental, es decir, se da de forma congénita aunque es susceptible de modificación por la influencia de factores extrínsecos, como la educación. En consecuencia, las conductas insertadas dentro de un perfil de déficit de atención a edades tempranas pueden considerarse como signos de alerta y en parte como variables predictoras de un futuro TDAH.

Se han obtenido pruebas de que las características temperamentales pueden definirse como innatas o emergentes durante el primer año de vida y de que son la base para la instauración de los principales rasgos de la personalidad en la edad adulta. Estos estudios avalan el concepto de estabilidad del comportamiento que propone Rutter (1987): «La permanencia de un proceso, estructura o mecanismo subyacente que se manifiesta de diferente manera según la edad». Si se toma como referencia esta concepción de estabilidad, puede considerarse que la potencialidad para atender es la misma en la infancia temprana que en la niñez.

Thomas y Chess ${ }^{4}$ definen el temperamento como «un término general que se refiere al cómo de la conducta. Difiere de la capacidad, que tiene que ver con el qué de la conducta y de la mejor o peor realización de ésta; también difiere de la motivación, que explica por qué hace una persona lo que hace». Sobre la base de su experiencia en el New York Longitudinal Study, estos autores proponen un modelo de temperamento con 9 dimensiones: nivel de actividad (componente motor presente en la actividad de un niño), regularidad (predictibilidad del ciclo sueño-vigilia, hambre, etc.), acercamiento o retraimiento (respuesta inicial a un nuevo estímulo: comida, juguete, persona, etc.), adaptabilidad (respuestas a situaciones nuevas), umbral de respuesta (intensidad del estímulo necesario para suscitar una respuesta), intensidad de reacción (grado de energía de la respuesta), humor (proporción de conductas agradables), tendencia a la distracción (grado en el que los estímulos irrelevantes interfieren en una actividad), atención (grado de concentración en cada actividad) y persistencia (matenimiento de la atención en una misma tarea durante un período de tiempo suficientemente extenso).

Varias de estas dimensiones temperamentales guardan relación directa con la atención y con la actividad motriz: el nivel de actividad se refiere a la cantidad de movimiento al bañarse, al comer, al jugar, al vestirse, etc; la tendencia a la distracción se refiere a la menor o a la mayor facilidad con que los estímulos ambientales interfieren en la dirección de la conducta; la atención y la persistencia se refiere a la 
cantidad de tiempo dedicado a una actividad (atención sostenida) y a la continuidad de ésta entre los obstáculos, respectivamente ${ }^{5}$.

Buss y Plomin ${ }^{6}$ describen el temperamento como «tendencias generales heredadas»; Sanson et $\mathrm{al}^{7}$ lo describen como «diferencias individuales de autorregulación atencional, emocional y conductual, junto con un grado relativo de reactividad emocional que dan un carácter único a un individuo»; Bates $^{8}$ describe que el temperamento «se configura según las diferencias individuales de tendencias conductuales y de raíz biológica que aparecen muy pronto en la vida y son relativamente estables en distintos tipos de situaciones y en el transcurso de la vida». Por su parte, McClowry ${ }^{9}$ identificó 4 componentes primarios del temperamento relativamente estables, entre los que se encuentran el período de atención y constancia (relacionado con la capacidad de mantener el esfuerzo y la atención) y la actividad (referida a la propensión a ocuparse en conductas con abundante movimiento) ${ }^{5}$.

Algunos autores afirman que el rasgo comportamental más estable de los 12 a los 24 meses es la atención ${ }^{10-12}$. Se parte de la hipótesis de que la estabilidad atencional es una dimensión nuclear del temperamento que se manifiesta de manera diferente durante la vida. Cabe esperar que los niños con baja capacidad atencional en la niñez tardía se comporten así desde la infancia temprana; en consecuencia, estos rasgos irregulares referentes a la concentración en edades tempranas pueden servir para predecir futuros perfiles disfuncionales de la atención y del autocontrol ${ }^{13}$.

En cambio, otros autores han estudiado un gran número de factores que influyen negativamente en la estabilidad del temperamento, entre los que se incluyen los siguientes: el entorno familiar y sociocultural, la consolidación de patrones de comportamiento, las experiencias de aprendizaje, la influencia del sexo y de la edad y el contexto socioeconómico. Así, el aprendizaje de hábitos puede influir en la manifestación de la capacidad atencional: a mayor adquisición de hábitos que favorecen la focalización y el mantenimiento de la atención durante la infancia temprana, menor expresión de conductas relacionadas con un perfil atencional alejado de la normalidad en la niñez ${ }^{13}$. Aunque hasta el inicio del segundo año ya han influido variables ambientes, a esta edad siguen teniendo mayor peso los factores biologicotemperamentales.

Son muchos los investigadores que han intentado analizar la constancia de los rasgos del temperamento desde la infancia hasta la edad adulta; esto no es fácil debido a la influencia de variables ambientales y educativas a través de los años. Caspi et $\mathrm{al}^{14}$ observaron que los niños de 3 años con un temperamento caracterizado por falta de control (corto mantenimiento de la atención, entre otras variables) manifestaban a la edad de 9 años dificultad para inhibir conductas impulsivas, permanecer en sus pupitres y persistir en las tareas. Sobre la base de sus tipologías en los datos de evaluación del Temperament Assessment Battery for Children (1999), Martin y Bridger ${ }^{15}$ descubrieron que las respuestas de padres y de maestros se traducían en 6 tipos, con estabilidad moderada, entre los que se encuentran el tipo impulsivo, que engloba dificultad para la autorregulación y el control ${ }^{5}$.

Los objetivos de esta investigación son los siguientes: describir el repertorio conductual a la edad de 12 a 15 meses de niños posteriormente diagnosticados de TDAH y encontrar variables predictoras en la infancia temprana de un desarrollo irregular de la atención. La finalidad última de este estudio es de naturaleza preventiva y educativa: justificar la necesidad del desarrollo de programas de asesoramiento parental y de atención temprana y guiar la intervención compensatoria. La hipótesis por contrastar es que los niños diagnosticados de TDAH en la edad escolar tienen atención lábil y escaso autocontrol, que eran ya evidentes al inicio del segundo año de vida.

\section{Sujetos y métodos}

El grupo clínico lo constituyen 50 sujetos (38 niños y 12 niñas) que se diagnosticaron de TDAH en la Unidad de Neurología Pediátrica de la Clínica Universitaria de Navarra con edades comprendidas entre 5 y 12 años. La distribución de este grupo por subtipos es la siguiente: el $70 \%$ del subtipo combinado $(n=35)$; el $22 \%$ del subtipo predominantemente inatento $(n=11)$ y el $8 \%$ del subtipo predominantemente hiperactivo e impulsivo $(n=4)$. Los componentes de este grupo presentan, en la escala de Wechsler, un cociente intelectual comprendido entre 83 y 134 (cociente intelectual medio de 105). El grupo control lo constituyen 30 sujetos de la población general del mismo rango de edad.

Para llevar a cabo esta investigación se utilizó como instrumento de medida el TBQ (Toddler Behaviour Questionnaire $)^{16-18}$. Hagekull ${ }^{14}$ diseñó este cuestionario para estudiar el temperamento de los niños de 12 a 15 meses a través de las respuestas de los padres sobre las conductas de sus hijos en la actualidad. Sin embargo, en este estudio los padres cumplimentaron de forma retrospectiva cuando acudían a la consulta. Para minimizar este sesgo se utilizaron las respuestas parentales referentes a los niños del grupo control, aunque es posible que los padres de los niños con problemas de conducta tiendan a sesgar los recuerdos del pasado de forma que sean consistentes con la conducta actual de su hijo. El TBQ se forma por 37 ítems sobre la conducta del niño en 7 áreas: sueño, comida, aseo y vestido, reacciones ante nuevos lugares, impresiones sensoriales, reacciones ante la gente y juego. Cada ítem consta de 2 afirmaciones que representan el máximo y el mínimo de intensidad o el máximo y el mínimo de frecuencia entre los que una determinada conducta se puede mostrar; exige puntuarse de uno a 5 según se asemeje la conducta del niño a un extremo o a otro (escala de diferencial semántico). Mediante la utilización de la escala de Brazelton ${ }^{19}$ para el período neonatal y mediante el TBQ para la edad de 12 meses, Poch ${ }^{18}$ comprobó la correlación entre los siguientes factores: regulación de la conducta, orientación hacia estímulos auditivos y visuales e interacción de uno y otro período.

Por otra parte, en la consulta clínica se cumplimentó con los padres la escala DSM-IV (Diagnostic and Statistical Manual of Mental Disorders 'Manual Diagnóstico y Estadístico de los Trastornos Mentales') para TDAH cuantificada, en función de las conductas que los niños afectados manifiestan en la actualidad. También los padres de los niños que constituían el grupo control respondieron a esta escala. De esta forma se obtuvieron 2 medidas del repertorio conductual de todos los participantes del estudio: a la edad de 12 a 
Tabla 1 Agrupaciones factoriales de los ítems del Toddler Behaviour Questionnaire, diferencias de medias entre los 2 grupos de la muestra (grupo con trastorno por déficit de atención e hiperactividad y grupo control) y correlaciones con los criterios del Manual Diagnóstico y Estadístico de los Trastornos Mentales IV

Análisis factorial. Medida de adecuación muestral de Kaiser-Meyer-Olkin: 0,544. Los 7 factores resumen el $60 \%$ de la variancia total y las cargas factoriales son superiores a 0,60
tStudent:

TDAH-

control
Correlación con criterios para TDAH del DSM-IV

Adaptación a lo novedoso

Ítem 8: nota y reacciona ante diferentes sabores y consistencias de las comidas

Ítem 15: manifiesta reacciones fuertes, positivas o negativas, cuando se le cambia y se le viste

Ítem 20: se adapta tras unos pocos minutos a un nuevo lugar o a una nueva situación Ítem 27: la primera reacción ante un adulto desconocido es enérgica (ríe o llora) Ítem 33: cuando se le da un nuevo juguete lo mira y juega con éste durante $15 \mathrm{~min}$ o más

Estabilidad de la conducta en el juego

Ítem 32: puede jugar y divertirse él solo durante media hora en el suelo, en el parque, mirar y jugar con los juguetes, con las manos, etc.

Ítem 35: puede estar sentado concentrado durante un tiempo largo (15 min o más) viendo la televisión o «leyendo» un cuento

Ítem 36: se mueve mucho mientras juega

Ítem 37: cuando juega con otra persona puede seguir con el mismo juego bastante tiempo (15 min o más), por ejemplo: jugar al balón, cosquillas, etc.

Reacción ante los cuidados

Ítem 12: la reacción inicial ante la primera comida es fuerte, ya sea positiva o negativa Ítem 13: se mueve mucho o patalea cuando se le cambia de pañal y se viste Ítem 16: mientras se baña, patalea, salpica, se mueve, etc.

Ítem 17: muestra su gusto o disgusto por el baño de forma enérgica

Ítem 21: reacciona de forma enérgica (llanto, asombro, etc.) ante sonidos estridentes Ítem 25: muestra reacciones fuertes (ríe, llora, etc.) ante personas familiares

\section{Excitabilidad}

Ítem 18: está de buen humor (ríe, sonríe, etc) mientras se le cortan las uñas Ítem 22: reacciona de forma enérgica (llanto, asombro, etc.) ante luces brillantes Ítem 23: fuerte reacción ante los movimientos rápidos de una persona nerviosa Ítem 26: la primera reacción cuando ve a un adulto desconocido es positiva (ríe, sonríe, etc.)

Ítem 30: generalmente está de buen humor cuando lo toma o le acompaña un adulto extraño

Ítem 31: después de que un extraño lo toma durante algunos minutos acepta permanecer solo en la habitación con esa persona

Humor

Ítem 5: muestra claramente que tiene hambre (llora, señala la comida, etc.)

Ítem 10: ríe o sonríe tras la comida

Ítem 11: está de buen humor cuando se le limpia la cara tras la comida

Ítem 14: está de buen humor cuando se le cambia o viste

Ítem 19: está de buen humor cuando se le pone la ropa para salir a la calle

Ítem 34: normalmente ríe y sonríe mientras juega

Regularidad

Ítem 1: va a dormir aproximadamente a la misma hora por la noche y a la hora de la siesta

Ítem 2: despierta más o menos a la misma hora por la noche y a la hora de la siesta

Ítem 3: cuando sus horas de sueño se ven alteradas por viajes, visitas, etc., se muestra quejoso, mimoso o más activo de lo normal

Ítem 4: tiene unas horas al día en que se muestra más despierto y activo

Ítem 6: quiere que lo alimenten aproximadamente a la misma hora todos los días

Ítem 7: toma la misma cantidad de alimento en cada desayuno, comida, merienda, etc.

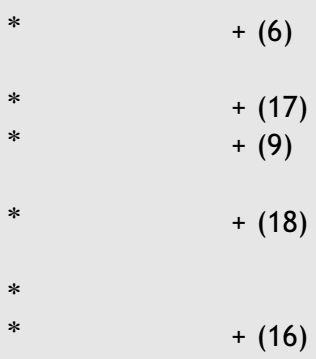

** 
Tabla 1 (continuación)

Análisis factorial. Medida de adecuación muestral de Kaiser-Meyer-Olkin: 0,544. Los 7 factores resumen el $60 \%$ de la variancia total y las cargas factoriales son superiores a 0,60
tStudent: TDAH-

control
Correlación con criterios para TDAH del DSM-IV

Ítem 9: se concentra mientras come y no se altera por lo que acontece alrededor

$* *$

Interacción social

Ítem 24: reacciona de manera distinta ante diferentes voces, por ejemplo, ante la voz de niños y de adultos

Ítem 28: reacciona de forma diferente cuando se encuentra frente a adultos o frente a niños

Ítem 29: nota claramente y reacciona de forma diferente ante las diferencias físicas en personas adultas, por ejemplo, el tipo de peinado, gafas, barba, etc.

+: correlación estadísticamente significativa; ( ): $\mathrm{n} .^{\circ}$ de criterios del Manual Diagnóstico y Estadístico de los Trastornos Mentales iv con los que se correlaciona; DSM-IV: Diagnostic and Statistical Manual of Mental Disorders 'Manual Diagnóstico y Estadístico de los Trastornos Mentales'; TDAH: trastorno por déficit de atención e hiperactividad.

$* p<0,01$.

$* * p<0,05$.

15 meses (a través del TBQ) y en la actualidad (a través de los criterios del DSM-IV).

Se realizó un análisis factorial de las puntuaciones de todos los sujetos en los ítems del TBQ para reducir el número de variables y facilitar las comparaciones entre los grupos y la interpretación de los resultados. Se utilizaron los métodos estadísticos de análisis de componentes principales y rotación de Varimax con Kaiser. Se obtuvieron 7 factores que se corresponden con los constructos siguientes (tabla 1): adaptación a lo novedoso (situaciones, comidas, lugares y juguetes nuevos y adultos desconocidos), estabilidad de la conducta en el juego (mantenimiento de la concentración en una misma tarea, actividad motriz durante el juego en solitario y actividad motriz durante el juego en compañía), reacción ante los cuidados (respuesta ante la comida, cambio de pañal, cambio de ropa, baño y personas que proporcionan los cuidados), excitabilidad (reacción ante estímulos fuertes: luces brillantes, movimientos rápidos, estímulos intrusivos y adultos desconocidos), humor (manifestación del estado de ánimo en situaciones relacionadas con la comida, el baño y el vestido), regularidad (ritmo en determinadas necesidades biológicas, como el sueño y la comida) e interacción social (reacción diferencial ante voces, características físicas y personas de edades diferentes).

\section{Resultados}

Al comparar las conductas registradas en el TBQ entre los grupos con TDAH y control, se observaron diferencias estadísticamente significativas en 12 de los 37 ítems del cuestionario (fig. 1 y tabla 1). Frente al repertorio de conducta observado en el grupo control, los pacientes afectados de TDAH a edad escolar presentaban, al inicio del segundo año de vida, conductas alejadas de la normalidad. Así, los niños de este grupo, según la percepción retrospectiva de sus padres, presentaban más dificultades para dormirse y despertarse a la misma hora, se

\begin{tabular}{|c|c|c|}
\hline TDAH $(\mathrm{N}=50)$ & & CONTROL $(\mathrm{N}=30)$ \\
\hline $\begin{array}{c}2^{\circ} \text { año de vida } \\
\text { (TBQ) }\end{array}$ & $\leftarrow$ t-Student $\rightarrow$ & $\begin{array}{l}2^{\circ} \text { año de vida } \\
\text { (TBQ) }\end{array}$ \\
\hline$\uparrow$ Correlación $\downarrow$ & & $\uparrow$ Correlación $\downarrow$ \\
\hline $\begin{array}{l}\text { Actualidad } \\
\text { (DSM-IV) }\end{array}$ & $\leftarrow$ t-Student $\rightarrow$ & $\begin{array}{l}\text { Actualidad } \\
\text { (DSM-IV) }\end{array}$ \\
\hline
\end{tabular}

Figura 1 Los grupos TDAH y control han sido evaluados en dos momentos diferentes: al inicio del segundo año de vida y en la actualidad, mediante el TBQ y los criterios DSM-IV respectivamente.

distraían más fácilmente con estímulos irrelevantes mientras se les daba de comer, tendían a quejarse más cuando se les aseaba, se les cambiaba el pañal y se les vestía, tenían dificultades para discriminar adultos y niños, cambiaban de ocupación tras pocos minutos de comenzar a realizarla en solitario o en compañía, se movían mucho durante el juego, no eran capaces de permanecer sentados durante un período de tiempo prolongado y cuando se les daba un juguete nuevo lo miraban y jugaban con él durante poco tiempo.

De esta manera, se manifiestan las diferencias en el repertorio conductual a la edad de un año entre niños sanos y niños diagnosticados de TDAH en la infancia tardía. A partir del sumatorio de las puntuaciones de estos 12 ítems, en los que se diferencian de forma significativa ambos grupos, se obtiene una nueva variable del TBQ creada ex novo; la puntuación en esta variable oscila entre 12 y 60 puntos (puesto que cada ítem se puntúa del uno al 5). En esta variable, que da información acerca de la diferencia entre ambos repertorios conductuales, el $56 \%$ de los niños afectados de TDAH obtuvo una puntuación igual o menor de 44 puntos, mientras que sólo el $3 \%$ de los niños del grupo control obtuvo una puntuación de esta forma. Esta diferencia no se debe al azar; por consiguiente, la obtención 
de una puntuación inferior a 44 puntos puede considerarse un signo de alerta. Por otra parte, esta variable se correlaciona de forma significativa $(p<0,01)$ con todos los criterios diagnósticos de TDAH del DSM-IV. Es decir, las diferencias halladas entre las conductas de los niños con TDAH y los niños del grupo control a la edad de un año parecen corresponderse con una diferencia en el perfil atencional de éstos en la infancia tardía. Estos resultados no permiten hablar de relación causal y, por consiguiente, no se puede atribuir a la variable diferencial la denominación de predictora, pero sí facultan para referirse a la obtención de una puntuación baja en ésta como un signo de alerta para tener en cuenta.

Se estudiaron las diferencias existentes entre los grupos TDAH y control en los 7 factores del TBQ obtenidos a partir del análisis factorial. Según se resume en la tabla 1 , se hallaron diferencias en 3 de estos factores $(p<0,05)$ : regularidad, humor y estabilidad de la conducta en el juego. Las medias de las puntuaciones de los niños con TDAH en estos factores fueron menores que las medias de los componentes del grupo control.

En cuanto a la dimensión regularidad, los sujetos con TDAH mostraban a la edad de 12 a 15 meses, frente al grupo control, mayor dificultad para conciliar el sueño y despertarse todos los días a la misma hora, no poseían unas horas al día en las que se mostraban más despiertos y activos y, cuando sus horas de sueño se alteraban, su conducta no cambiaba. También mostraban una tendencia más acusada que la de los niños del grupo control a tener hambre a diferentes horas según el día, a no tomar la misma cantidad de alimento en cada comida y a distraerse fácilmente con lo que ocurría mientras comían.

En cuanto a la dimensión humor, los niños con TDAH, frente a los del grupo control, mostraban de forma menos enérgica sentirse hambrientos, no tendían a sonreír tras ser alimentados pero sí a quejarse cuando se los aseaba o vestía y no tendían a reír mientras jugaban.

En cuanto a la dimensión estabilidad de la conducta durante el juego, se encontró que los niños con TDAH, frente al grupo control, mostraban menos capacidad de llevar a cabo una misma actividad durante un período de tiempo extenso al jugar solos y al jugar acompañados, así como de permanecer sentados, y se movían más mientras jugaban.

Por otra parte, como puede verse en la tabla 1, se encontraron diferencias estadísticamente significativas entre los niños afectados de TDAH del subtipo inatento y los niños del subtipo combinado en 2 de los 7 factores hallados en esta investigación $(p<0,05)$ : adaptación a lo novedoso y estabilidad de la conducta durante el juego. Respecto a lo novedoso, los pacientes con TDAH combinado al inicio del segundo año de vida reaccionaban peor que los pacientes del subtipo inatento ante nuevos sabores y consistencias de comidas y cuando se los cambiaba o vestía, se adaptaban peor a lugares nuevos y tenían reacciones más fuertes ante los adultos desconocidos. Respecto al juego, el grupo con TDAH combinado frente al grupo con TDAH inatento presentaba mayor incapacidad para seguir con la misma actividad durante mucho tiempo al jugar con otra persona, tenía mayor dificultad para jugar y divertirse en solitario así como para seguir con una misma actividad durante un tiempo largo al jugar con otra persona, manifestaba mayor incapacidad para permanecer sentado durante un período de tiempo dilatado y mayor tendencia a moverse durante el juego.

Se analizó la correlación entre los ítems del cuestionario TBQ y los criterios del DSM-IV para TDAH a partir de las puntuaciones de todos los sujetos de la muestra (fig. 1). Se observa que 6 ítems del TBQ se correlacionan significativamente con 6 o más criterios de los 18 del DSM-IV para TDAH. En concreto, el ítem «puede estar sentado concentrado durante un tiempo largo viendo la televisión o "leyendo" un cuento» se correlaciona con todos éstos y el factor estabilidad de la conducta durante el juego se correlaciona con 9 criterios de inatención y 8 criterios de hiperactividad e impulsividad (tabla 1). Este dato demuestra que el factor juego en la infancia temprana además de ser diferente en los niños con TDAH y en los niños del grupo control guarda relación con el perfil atencional de éstos en la infancia tardía. En efecto, al analizar los ítems que componen este factor se observa que todos tienen relación directa con la manifestación de la atención a la edad de un año: la capacidad de permanecer ocupado con la misma actividad durante un período de tiempo extenso cuando juega solo y cuando juega acompañado, la capacidad de estar sentado concentrado con un mismo juguete durante un tiempo largo y la cantidad de movimientos que lleva a cabo cuando juega.

\section{Discusión}

Durante los últimos años ha sido creciente el interés por el estudio de los rasgos de TDAH a edad preescolar ${ }^{20}$. En esta investigación se intentó remontar la perspectiva hasta un período más temprano, el inicio del segundo año, ya que se trata de buscar el anclaje del trastorno en variables temperamentales.

Respecto a la herencia del temperamento, hay cierto consenso en torno a que los rasgos temperamentales, en especial los que corresponden a atributos negativos, como la proclividad al miedo o al enfado, reciben una «moderada influencia genética» ${ }^{21}$. Al analizar las valoraciones de los padres de gemelos idénticos y fraternos y de sus hermanos, Matheny ${ }^{22}$ mostró importantes efectos de la herencia en 5 de las 9 dimensiones temperamentales de Thomas y Chess ${ }^{4}$ : acercamiento-retraimiento, adaptabilidad, umbral de respuesta, actividad y atención.

Estudios longitudinales han obtenido datos a favor de la estabilidad de la capacidad atencional durante la infancia temprana con correlaciones significativas entre los rendimientos de los mismos niños en tareas de atención selectiva a la edad de $18,24,30$ y 36 meses $^{23-27}$. Por otra parte, se demuestra que la capacidad atencional influye en otras dimensiones del desarrollo. Algunas investigaciones señalan que, al año de vida, hay relación entre la atención sostenida y el aprendizaje de contingencias y de vocabulario en niños que no presentan un trastorno específico del lenguaje ${ }^{28,29}$.

Varias investigaciones muestran pruebas de la estabilidad de la atención sostenida durante el juego en el período comprendido entre los 7 y los 12 meses de edad ${ }^{30}$, del primer al segundo año de vida ${ }^{31}$ y de los 6 meses a los 2 años $^{32}$. En cuanto a la posible predicción de síntomas de inatención, se encuentra una correlación significativa entre la atención sostenida a la edad de 2 años y a la de 3 años y medio $^{33}$. Por otra parte, se ha validado una escala de 
observación de las conductas de niños de 30 meses y se ha obtenido que los atributos relacionados con el mantenimiento de la atención tienen una validez predictiva de 6 meses ${ }^{20}$. Putnam et $\mathrm{al}^{26}$ han manifestado la consistencia de las respuestas de los padres acerca de los comportamientos de sus hijos a la edad de 24 y 36 meses y han obtenido correlaciones estadísticamente significativas.

En un estudio reciente ${ }^{34}$ acerca de las percepciones de maestras de guarderías acerca de las conductas de los niños que están a su cargo se concluye que hay rasgos comportamentales a edades muy tempranas que pueden ser manifestación del TDAH, como no prestar atención a la maestra, no participar en juegos colectivos, dificultad para seguir instrucciones y mostrar respuestas muy fuertes (quejas y llantos incesantes) al sentirse incómodos o cuando no consiguen lo que quieren. Gimpel y $\mathrm{Kuhn}^{35}$ emplearon los criterios DSM-IV para TDAH y encontraron que en Nebraska y Utah uno de cada 10 niños de edades comprendidas entre 2 y 6 años muestra rasgos de inatención o de hiperactividad e impulsividad.

En la interpretación de los resultados obtenidos deben tenerse en cuenta algunas limitaciones de este estudio. En primer lugar, se investigan las percepciones retrospectivas de los padres de niños de edades comprendidas entre 5 y 12 años diagnosticados de TDAH acerca de las conductas de sus hijos cuando tenían entre 12 y 15 meses. Al contar con un grupo control constituido por sujetos de la población general del mismo rango de edad cuyos padres informan también retrospectivamente acerca de su conducta en el segundo año, se corrige el citado sesgo. No obstante, conviene tener en cuenta la posible tendencia de los padres de los niños con dificultades a sesgar sus recuerdos acerca de los comportamientos de sus hijos en el pasado, de forma que sean consistentes con la conducta actual. Por otra parte, al conocer los investigadores el estatus paciente/control de los sujetos de la muestra, no se controló el sesgo del evaluador. Por último, los análisis estadísticos que se llevaron a cabo en este trabajo permiten conocer la relación existente entre el estilo comportamental en lo referido a la atención a la edad de 12 a 15 meses y el estilo comportamental mostrado en la edad escolar, pero no facultan para hablar de causalidad.

Como se explicó en el apartado Resultados, las principales diferencias halladas entre los sujetos con TDAH y los sujetos del grupo control pueden agruparse en 3 factores: regularidad, humor y estabilidad de la conducta en el juego. En el factor humor se observó que los niños afectados de TDAH no tendían a manifestar su bienestar con una sonrisa después de alimentarse ni a reír mientras jugaban, aunque sí expresaban su malestar cuando se les cambiaba el pañal, bañaba o vestía. Por otra parte, en comparación con el grupo control, los niños afectados de TDAH se quejaban menos cuando tenían hambre y durante el juego se movían en exceso y cambiaban de actividad con mucha frecuencia. Por último, los hallazgos de esta investigación indican que los sujetos escolares del grupo con TDAH habían mostrado, a la edad de 12 a 15 meses, baja regularidad en sus hábitos básicos (comida y sueño).

Como se ha descrito, se encontró que 6 ítems del TBQ se correlacionaban de forma significativa con 6 o más criterios del DSM-IV para TDAH. Como puede apreciarse en la tabla 1, el factor estabilidad de la conducta durante el juego se correlaciona con 9 criterios de inatención y 8 criterios de hiperactividad e impulsividad y el ítem referente a la capacidad para permanecer sentado durante un período de tiempo amplio viendo la televisión o «leyendo» un cuento se correlaciona con los 18 criterios del DSM-IV.

En conclusión, este estudio aporta suficientes indicios de que los niños afectados de TDAH en edad escolar, considerados como grupo, mostraban ya al principio del segundo año rasgos de inatención y de escaso autocontrol. De esta forma, se confirma la hipótesis de que la atención y la regulación de la conducta son dimensiones básicas del estilo comportamental, altamente influidos por factores biológicos. Este estudio debería seguirse con otros de carácter prospectivo con el objeto de realizar aportaciones al ámbito de la educación temprana, preventiva y compensatoria.

Para guiar el desarrollo de programas de atención temprana e intervención compensatoria, se proponen algunas pautas. En el área de regularidad, asignar una hora fija para acostar al niño por la noche y a la hora de la siesta así como para cada una de las comidas: evitar que ninguna otra actividad o estímulo distractor interfiera en el hábito de sueño y de comida, y tratar de que formen parte de una rutina (seguir siempre los mismos pasos, del mismo modo, en los mismos lugares, etc.). En el área del humor, se propone reforzar, mediante el uso de gratificaciones preferentemente no materiales, las conductas que quieran aumentarse o instaurarse (como la sonrisa o la risa mostradas durante el juego o tras ser alimentados, aseados, premiados, etc.) y emplear técnicas (como, por ejemplo, la retirada de atención) para disminuir o paliar los comportamientos desadaptados cuando se los asea o se les administra cualquier otro tipo de cuidados: quejas, llantos, gritos, etc. En el área de la estabilidad de la conducta en el juego, conviene darles los juguetes de uno en uno (evitar así la famosa «cesta de los juguetes») y espaciados en el tiempo para que puedan dedicarse a una misma actividad de forma prolongada; asimismo, se aconseja acostumbrarlos a jugar sentados o a jugar en espacios reducidos a fin de evitar que se muevan constantemente o sin un fin instrumental así como a jugar solos en ocasiones con el objetivo de que no dependan de otras personas para entretenerse y divertirse.

\section{Finaciación}

La Fundación Fuentes Dutor (ICT) y la ADA de la Universidad de Navarra subvencionaron este trabajo. Beca-premio de la Sociedad Española de Neurología Pediátrica (SENP), septiembre de 2008.

\section{Bibliografía}

1. Purper-Ouakil D, Cortese S, Wohl M, Asch M, Acquaviva E, Falissard B, et al. Predictors of diagnostic delay in a clinical sample of French children with attention-deficit/hyperactivity disorder. Eur Child Adolesc Psychiatry. 2007;16:505-9.

2. Ralston SJ, Lorenzo MJ. ADORE -Attention-Deficit Hiperactivity Disorder Observational Research in Europe. Eur Child Adolesc Psychiatry. 2004;13:36-42.

3. Taylor E, Dopfner M, Sergeant J, Asherson P, Banaschewski T, Buitelaar J, et al. European clinical guidelines for hyperkinetic disorder-first upgrade. Eur Child Adolesc Psychiatry. 2004; 13(Suppl 1):7-30. 
4. Thomas A, Chess S, editors. Temperament and development. New York: Brummer/Mazel; 1997.

5. Keogh B, editor. Temperamento y rendimiento escolar. Madrid: NARCEA, S.A; 2006.

6. Buss $\mathrm{AH}$, Plomin $\mathrm{R}$, editors. A temperament theory of personality. New York: John Wiley \& Sons; 1975.

7. Sanson AV, Smart DF, Prior M, Oberklaid F, Pedlow R. The structure of temperament from age 3 to 7 years: Age, sex, and sociodemographic influences. Merrill Palmer Q. 1994;40: 233-52.

8. Bates JE. Applications of temperament concepts. En: Kohnstamm GA, Bates JE, Rothbart MK, editors. Temperament in childhood. Chichester: John Wiley \& Sons, Ltd; 1989. p. 321-55.

9. McClowry SG. The development of the School-Age Temperament Inventory (SATI). Merrill Palmer Q. 1995;41:271-85.

10. Kohnstamm GA, Bates JE, Rothbart MK, editors. Temperament in childhood. Chichester: John Wiley \& Sons; 1989.

11. Nichols KE, Fox N, Mundy P. Joint attention, self-recognition, and neurocognitive function in toddlers. Infancy. 2005;7:35-51.

12. Claussen AH, Mundy PC, Mallik SA. Joint attention and disorganized attachment status in infants at risk. Dev Psychopathol. 2002;14:279-91.

13. Brown JH, Johnson MH, Paterson SJ, Gilmore R, Longhi E, Karmiloff-Smith A. Spatial representation and attention in toddlers with Williams syndrome and Down syndrome. Neuropsychologia. 2003;41:1037-46.

14. Caspi A. Personality development across the life course. En: Damon W, editor. The handbook of child psychology. New York: John Wiley \& Sons; 1989. p. 311-75.

15. Martin RP, Bridger RC, editors. The temperament assessment battery for children-revised. Athens: University of Georgia; 1999.

16. Hagekull B. The baby and toddler behavior questionnaires: Empirical studies and conceptual considerations. Scand J Psychol. 1985;26:110-22.

17. Poch ML. Desarrollo cognitivo e interactivo durante el primer año de vida: estudio mediante las escalas de Brazelton, de Hagekull y de Uzgiris-Hunt. Tesis doctoral. Pamplona: Universidad de Navarra; 1991.

18. Poch ML, Narbona J. Valoración del "estilo comportamental" durante el primer año de vida. Act Ped Esp. 1994;52:70-6.

19. Brazelton TB, editor. Neonatal Behavioral Assessment Scale (NBAS). London: SIMP and Blackwell; 1984.

20. Deutscher B, Fewell RR. The development and use of the attention deficit hyperactivity disorder-observational rating scale: Factor analysis and a preliminary investigation of predictive validity. J Psychoeducat Asses. 2001;19:317-29.

21. Goldsmith HH, Lemeny KS, Aksan N, Buss KA. Temperament substrates of personality development. En: Molfese VJ, Molfese
DL, editors. Temperament and personality development across the life span. Mahwah: Lawrence Erlbaum Associates; 2000. p. 1-32.

22. Matheny Jr AP. Temperament and cognition: Relations between temperament and mental test scores. En: Kohnstamm GA, Bates JE, Rothbart MK, editors. Temperament in childhood. Chichester: John Wiley \& Sons, Ltd; 1989. p. 263-82.

23. Van Aken C, Junger M, Verhoeven M, Van Aken MAG, Dekovic M. The longitudinal relations between parenting and toddlers' attention problems and aggressive behaviours. Infant Behav Dev. 2008;31:432-46.

24. Shaw DS, Lacourse E, Nagin DS. Developmental trajectories of conduct problems and hyperactivity from ages 2 to 10 . J Child Psychol Psychiatry. 2005;46:931-42.

25. Zhou Q, Hofer C, Eisenberg N, Reiser M, Spinrad TL, Fabes RA. The developmental trajectories of attention focusing, attention and behavioural persistence, and externalizing problems during school-age years. Dev Psychol. 2007;43:369-85.

26. Putnam SP, Gartstein MA, Rothbart MK. Measurement of finegrained aspects of toddler temperament: The early childhood behavior questionnaire. Infant Behav Dev. 2006;29:386-401.

27. Miller E, Joseph S, Tudway J. Assessing the component structure of four self-report measures of impulsivity. Pers Indiv Differ. 2004;37:349-58.

28. Aksan N, Kochanska G. Links between systems of inhibition from infancy to preschool years. Child Dev. 2004;75:1477-90.

29. Dixon Jr WE, Salley BJ, Clements AD. Temperament, distraction, and learning in toddlerhood. Infant Behav Dev. 2006;29: 342-57.

30. Lawson KR, Ruff HA. Focused attention: Assessing a fundamental cognitive process in infancy. En: Singer LT, Zeskind PS, editors. Biobehavioral assessment of the infant. New York: Guilford Press; 2001. p. 293-311.

31. Lawson KR, Ruff HA. Early attention and negative emotionality predict later cognitive and behavioral function. Int J Behav Dev. 2004;28:157-65.

32. Heinicke CM, Diskkin SD, Ramsey-Klee DM, Oates DS. Pre- and postbirth antecedents of 2-year-old attention, capacity for relationships and verbal expressiveness. Dev Psychol. 1986;22: 777-87.

33. Ruff HA, Lawson KR, Parrinello R, Weissberg R. Long-term stability of individual differences in sustained attention in the early years. Child Dev. 1990;61:60-75.

34. Hong Y. Teachers' perceptions of young children with ADHD in Korea. Early Child Dev Care. 2007;178:399-414.

35. Gimpel GA, Kuhn BR. Maternal report of attention deficit hyperactivity disorder symptoms in preschool children. Child Care Health Dev. 2000;26:163-79. 